\title{
EXAMINING THE TENSION CHORDING PRINCIPLE FOR A BEAM UNDER TORSION LOAD
}

\author{
E. S. Uttich ${ }^{凶}$, M. Bartz and B. Bender \\ Ruhr-Universität Bochum, Germany \\ $\triangle$ uttich@lpe.rub.de
}

\begin{abstract}
Tension chording is a lightweight design principle in the human motion system. More muscles than necessary are available in this system to generate motion. By using these redundant muscles the principle contributes to the lightweight design of the motion segments. The lightweight design benefits of the principle for technical structures loaded with bending torques were shown in prior studies. This paper presents a pilot study on lightweight design benefits of using tension chording for torsion loaded structures.
\end{abstract}

Keywords: lightweight design, biologically inspired design, biomimicry (biomimetics)

\section{Introduction}

In the context of increasing demands on energy and resource efficiency of technical products, technical lightweight design plays an important role in product development to put sustainability goals into practice (the Press and Information Office of the Federal Government 2018). Lightweight design is a design principle intended to minimize structural mass without diminishing the structure's functions, which is possible by completely using the structure's load-bearing capacity. This principle can lead to energy savings, but also to increased design and manufacturing costs. To find a compromise between cost and value is a central issue in the development of technical lightweight designs (Wiedemann, 2007; Klein, 2013). Lightweight design can also be found in nature, where biological designs are always subject to selection pressure which enforces solutions that are well-adapted to their environmental conditions (Degischer and Lüftl, 2009; VDI 6220-1, 2019).

An often successfully used source of inspiration for technical lightweight design is the human locomotive system. The lightweight design of the human locomotive system results from the interplay of different principles. One material-related lightweight design principle is the composite construction of the bone called hierarchical bone structure (Weiner and Wagner, 1998). Additionally, the lightweight design of the human locomotive system mainly results from the interaction of two principles: (1) Functional adaptation describes the adaptation bone mass along main tension paths. (2) Tension chording names the usage of ligaments and muscles to generate target movements and solving the hyperstatic system caused by redundant muscles to minimize bending in the bone during movement generation (Pauwels, 1965; Neilson, 1993; Scott, 2004). The interaction of these lightweight design principles results in a bone geometry in which bending stress is minimized. By minimizing the bending stress, its variation is homogenized over time and along the bone axis. Consequently, mass and resources can be saved (Pauwels, 1965; Mattheck, 1997). As an example for the tension chording principle, moving a load by flexing the arm is shown in Figure 1. All of the five different 
muscles displayed can be used to achieve the movement. To decide which muscles should be used for the movement can be considered an optimisation problem, which is described in more detail in section 2.1. As stated before, one optimization criterion to be considered is bending in the bones, which is shown for different muscle activities in Figure 1. Solving the problem for minimal bending load leads to decreased stress in the structure. This stress decrease combined with functional adaptation leads to decreased bone mass.
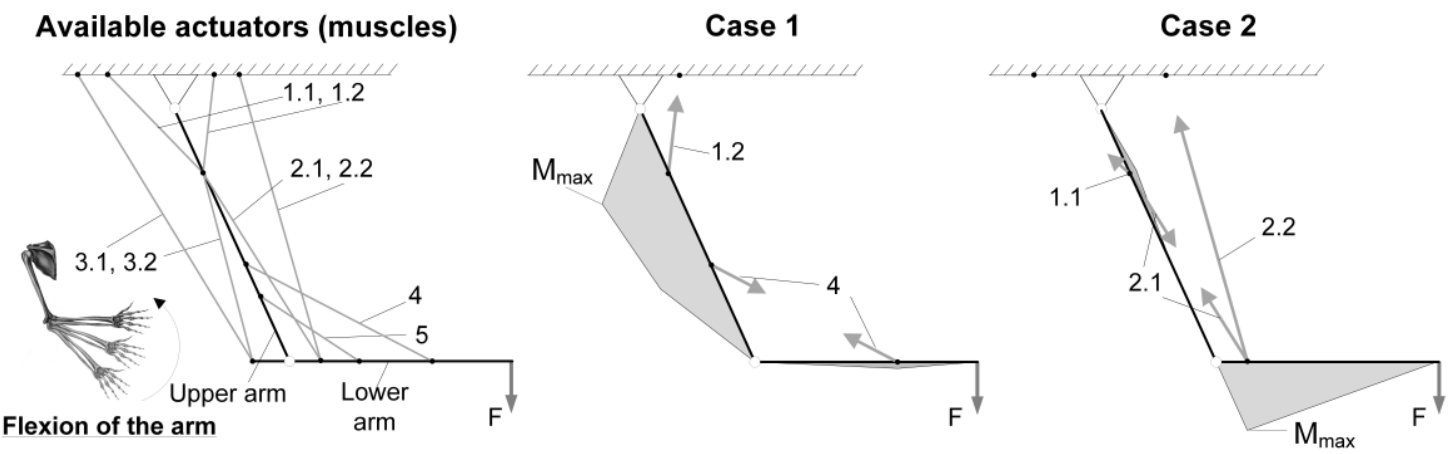

Figure 1. Redundant motion generation for the flexion of the arm in analogy to an example by Richard and Kullmer (2014). Figure adapted from Figure 2 in Bartz et al. (2018a)

Some of the human locomotive system's lightweight design principles are already technically applied. However, designers do not consider all aspects in the application of the principles. Therefore, lightweight design principles of the human locomotive system hold the potential for the optimization of technical products. One of these principles that is not yet used to its full potential is tension chording. Designers can use it to reduce bending in the structure by redundant actuators. So far, tension chording is investigated for structures loaded with one-dimensional bending. In prior studies, technical lightweight design potentials of tension chording for this load case were shown (Bartz et al., 2018a; Bartz et al., 2018b; Bartz et al., 2019). Possible areas of application for tension chording are presented in a prior study (Uttich et al., 2019), but systems in the identified application scenarios are subject to more loads but bending, e.g. to torsion. We want to investigate if it might also be possible to reduce these additional loads with the tension chording principle.

In this paper, we investigate the lightweight design potential of tension chording for torsion and its superposition with bending on a beam. It is of particular interest if this principle results in a weight saving compared to a beam with fixed support. Another point of interest is the influence of the angle between the tension chords and the beam on the result. To answer these questions, we structured the paper as follows. In section 2, we start explaining the effect of tension chording to a higher level of detail, focusing on the human body first, and elaborating technical applications then. Section 3 contains the experimental and simulative setup, including assumptions and the load-bearing structure. Last, in section 4, we discuss the results of the simulation and draw conclusions with regard to weight saving potential compared to previous investigations.

\section{Transfer of tension chording from nature to technology}

Transferring and using the lightweight design principles tension chording and functional adaptation from the human locomotive system to technology is not a new idea. Designers and researchers use functional adaptation in various technical processes for computer-assisted structural optimization of technical components. Examples of applications are Soft-Kill Option and Computer-Aided Optimization (Mattheck, 1997; VDI 6224-3, 2017), Finite Element Structural Synthesis (Witzel and Preuschoft, 2005) and processes for technical composite materials such as Reuschel (1999) and Völkl et al. (2017). These methods are based on algorithms simulating stress-controlled material remodeling and thus enabling a synthesis of component structures suitable for stress. The lightweight design principle tension chording is applied in a simple manner to dynamic constructions, e.g. bionic robots. In most applications, passive and active tension chord elements are used to generate motion and damping while causing minimal bending stress (Witte et al., 2000; Möhl, 2003; Klug et al., 2005; Ananthanarayanan et al., 2012). In contrast to the biological model, the potential of the lightweight 
design principle tension chording is not used to its full extend in these applications. Further potential lays in including the muscle redundancy principle, as the designers of the technical systems only use a single force to minimize external bending loads without active control. Therefore, the positive effects of the interplay of the principles by reducing bending loads is only used partly yet. Our previous work, e.g. Bartz et al. (2019), starts at this point. With insights from the human locomotive system, we investigate the tension chording principle further for technological systems by using a biomimetic development process.

A biomimetic development process consists of three steps: analyzing a biological system, abstracting the system to a model and applying the model to develop a solution or a product. (VDI 6220, 2019). Bartz et al. (2019) investigated tension chording in combination with functional adaptation in the context of a biomimetic process and the focus on minimizing bending. As a result, we achieved findings on the tension chording principle along with a technical system that implements this principle and utilizes synergy with topology optimization methods. We could show that we can save mass by exploiting further aspects of the principle. Nevertheless, the scope of application of the findings and the technical principle is still limited to simple application scenarios. Therefore, we initiate a new biomimetic process with the aim of expanding the scope of application. In order to understand our approach better, we begin with explaining the effect of tension chording in more detail.

\subsection{Tension chording in the human locomotive system}

Tension chording is a lightweight design principle that utilizes functional integration for the actuation of kinematic chains to influence the system of loads acting on the chain. Loads that have a negative influence on the system of loads are converted into loads that are more favourable by using actuation loads that also fulfil another function. For example, loads that lead to bending are converted to normal loads by an additional force that introduces a reverse bend and also generates a part of the needed movement. Due to the reduced bending load, less bone material is required, which improves the use of material and resources (Pauwels, 1965). Possible outcomes for technical systems depend on system, goal and use case. For example, the structure's mass can be reduced by also using functional adaptation, higher loads can be moved, or higher accelerations are possible. The outcomes are accomplished by redundant actuation with linear actuators, whose activity is optimized for the system of loads. Linear actuators are actuators that act with a force on a structure. Examples are (artificial) muscles, hydraulic cylinders and belts. Redundant actuation means that there are more actuators available to generate motion of a structure than are actually needed, which is a hyperstatic system. For the human locomotive system, we call this optimization problem the muscle redundancy problem (Neilson, 1993; Scott, 2004). We are using Figure 1 to explain the whole idea with an example of a simplified human arm.

On the left of Figure 1, the flexion of the arm is shown, which is loaded by the external force $F$. In simplified terms, the flexion movement can be performed by the action of five muscles (1.1 to 5) shown in the mechanical model on the left side of the figure. Muscles can have more than one attachment point on bones. To take account for muscles with different attachment areas, muscles 1, 2 and 3 are modelled with two attachment points each. For the movement of the arm more muscle forces are available than are needed. The muscular forces can interact in different configurations to produce the same flexion movement. With case 1 and 2, two possible cases of muscle interaction are shown. In case 1, the flexion of the arm is primarily made possible by muscles 1 and 4, which produces a low bending load in the forearm, but a high bending load in the upper arm. In case 2, the movement is mainly generated by forces 1 and 2 , which minimizes the bending load in the upper arm, but increases the bending load in the forearm.

In accordance with the efforts of the musculoskeletal system to minimizing bending loads, both cases of movement generation shown in Figure 1 are not optimal. The human sensorimotor system is responsible for finding the optimal interaction of muscles, setting the number of muscles and the intensity of their activation for the movement. Part of finding the optimal interaction is not only to avoid unfavorable lever ratios and accelerations leading to bending loads. Moreover, an interaction of the muscles can be found in which the bending load on the bone is minimized (Pauwels, 1965). As bones cannot be remodelled to large extends by functional adaptation under changing loads, the interaction must also find muscle forces with which the bone geometry can remain similar. With this knowledge, we attempted to apply the principle to two technical systems under defined boundary conditions. 


\subsection{Tension chording and technological applications}

In order to transfer the tension chording principle to technology, we used a simple example in a pilot study on possible benefits of the principle before investigating examples that are more complex. For this purpose, we examine a beam articulated about the z-axis and actuated with a tension chord (Bartz et al., 2018a). We model the tension chord as a point force at the symmetry axis of the beam. The results show a reduction in volume to approx. $2.5 \%$ of the volume of a system with fixed support under the same load (Bartz et al., 2018a). Subsequently, we examined the principle for a more complex example and chose a robot arm with simplified boundary conditions (Bartz et al., 2019). The result of the transfer is displayed in Figure 2B. For this example, the tension chording makes it possible to reduce the mass of the dynamically moving parts of the robot arm by approx. $40 \%$ compared to a reference system (Figure 2A) without tension chording. To conclude, we can also save mass by using the tension chording principle for more complex examples, but mass savings are less than for the simplified system.
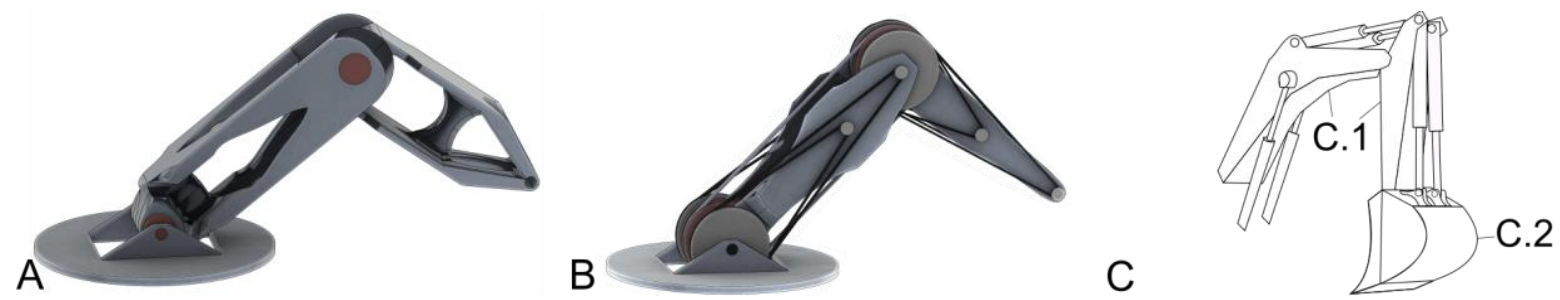

Figure 2. Transfer of tension chording to a simplified robot system (A and B): Reference system (A) and biomimetic tension chording system (B). Boom (C.1) and drag (C.2) of an excavator (C)

The applications for which we transferred the principle so far are still simple and do not allow an immediate utilization for real-life technical applications yet. For example, we optimized the robot arm for only one motion sequence. Therefore, we are going to transfer the principle to an applicationoriented system as the next step. For this purpose, we might have to extend the specification of the principle. In the course of a previous investigation, areas of application for tension chording were determined (Uttich et al., 2019). One possible application of the principle is an excavator.

An excavator roughly consists of a body, a boom and an attachable tool. The body includes, for example, the driver's cab and the hydraulic power packs of a hydraulic excavator. Figure $2 \mathrm{C}$ shows a hydraulically driven boom (C.1) with two segments and a drag (C.2) as an attachable tool. The boom segment connected to the body is hereafter referred to as segment 1 . If, for example, the right canine tooth of the drag as shown is engaged more strongly than the left canine tooth during soil removal, torsion occurs. This torsion is transferred from the drag suspension via the boom to the body.

Hydraulic cylinders can be understood as linear actuators or tension chords in the context of the tension chording principle. If the already redundant cylinders exert different forces on the structure, a torsional moment is generated. Instead of letting both cylinders act with the same force, they could be used to reduce torsion. The torsion resulting e.g. from the right canine tooth can be reduced by a counter pressure of the right cylinder. In this case, the right cylinder will exert a greater force than the left cylinder. Both are supported on segment 2 (C.1 right) near the joint to segment 1 (C.1 left). The torsion is thus transferred from the backhoe bucket to the support point via the actuators instead of the structure. There, however, further cylinders are attached, which transmit the torque to the middle of segment 1 , where it is transmitted via further cylinders into the body. The torsional moment could thus be transmitted via the actuators outside the boom system. While the given positioning and number of actuators is not sufficient to further reduce bending by using actuation redundancy, the question arises as to whether the tension chording principle can be used to advantage for systems with torsion.

\section{Experimental setup}

Before we investigate complex systems that have specific boundary conditions in the future, such as the boom presented in Figure 2C, we conduct a pilot study for a simple system in this paper. The object of investigation are possible benefits from using the principle to minimize torsion. Since many factors influence the potential benefit of the principle, the scope of the study is limited to a manageable number 
of factors. The first insights gained from the study help to decide which effort of investigating the principle to a higher level of detail is worthwhile for examples that are more complex.

\subsection{A load-bearing structure for one-dimensional structural optimization}

In Figure 3A, the load-bearing structure of the example system is displayed. It is a beam with a circleshaped cross-section area. $T$ radial lines separate and frame the beam into cylinders of height $h$ and diameter $d_{\mathrm{i}}$. The height is calculated by dividing the beam length $l_{\mathrm{x}}=1 \mathrm{~m}$ by the number of radial lines $T=100$. For each cross-section area, normal and shear force as well as bending and torsion moments are calculated. These forces and moments are used to calculate the minimal diameters needed to endure the loads at the cross-section area. In order to account for varying loads between the radial lines, a cylinder of diameter $d_{\mathrm{i}}$ is positioned at half-height of the cylinder on radial line $i$. As a result, cylinders of half height $(h / 2)$ are starting at the first and last radial line. 0B displays two variants of the tension chord attachment to the structure $\left(Z_{1}\right.$ with $Z_{2}$ and $Z_{1}$ with $Z_{2}$ ).

A.

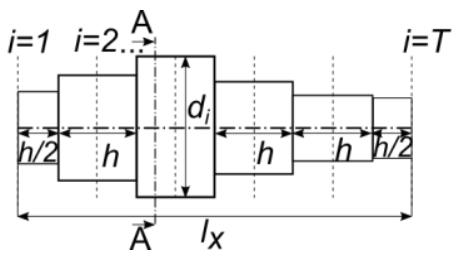

A-A

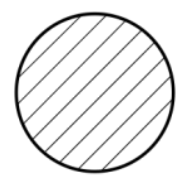

B.

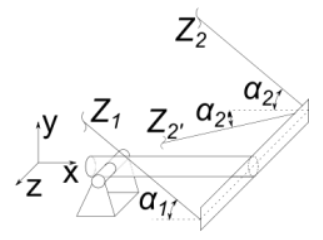

Figure 3. A. Separation of the beam into cylinders of height $h$ for volume optimisation. B. Beam with hinged support and lever arms for torsion compensating tension chords $Z_{1}$ and $Z_{2}$

The volume of the structure is calculated with Equation (1) where $n=T$.

$$
V=\left(\frac{d_{1}^{2}+d_{n}^{2}}{2}+\sum_{i=2}^{n-1} d_{i}^{2}\right) \cdot \frac{h \cdot \pi}{4}
$$

To calculate a load-dependent diameter for every cylinder-segment, von Mises yield criterion is used. The form for a beam with circular cross-section area is given in Equation (2). We take the absolute values of normal force $N_{\mathrm{x}}$ and bending moments $M_{\mathrm{by}}$ and $M_{\mathrm{bz}}$ to use the cross-section location with the highest load in every case. Shear forces in y and z direction are named $Q_{\mathrm{y}}$ and $Q_{\mathrm{z}}$, torsion moment is called $\mathrm{M}_{\mathrm{Q}}$.

$$
\sigma_{V}=\sqrt{\left(\frac{4 \cdot\left|N_{x}\right|}{\pi \cdot d^{2}}+\frac{32 \cdot\left(\left|M_{b y}\right|+\left|M_{b z}\right|\right)}{\pi \cdot d^{3}}\right)^{2}+3 \cdot\left[\left(\frac{16 \cdot M_{Q}}{\pi \cdot d^{3}}\right)^{2}+\left(\frac{16 \sqrt{Q_{y}{ }^{2}+Q_{z}}}{3 \cdot \pi \cdot d^{2}}\right)^{2}\right]}
$$

Solving this equation for the diameter $d$ leads to a polynomial of degree 6 . Numerical methods are used during the simulation to solve this polynomial for the maximal real value and $\sigma_{\mathrm{v}}=210 M P a$. We use this approach as a one-dimensional structural optimization. As we assume the density of the beam as homogenous, an optimization of the volume is equivalent to the optimization of the mass.

\subsection{Structural support, load cases and actuation variants}

Structural support is located on the left end of the beam. Two cases are part of the investigation: fixed support and hinged support with one degree of freedom about the z-axis. The fixed support serves as the basis for comparison. No actuation is used in this case. One key aspect of tension chording is functional integration. By providing one degree of freedom about the z-axis, the actuators can be used to move the beam about the axis and to compensate critical loads at the same time. Therefore, hinged support is used with the different tension chording variants. Please note that, for the investigation, the target movement about the $\mathrm{z}$-axis is zero. Figure 4 contains the two structural support variants as well as all load cases and actuation variants included in the investigation. The beam is represented as a line. At the right end of the line, a vertical line is displayed in the $\mathrm{x}$-z-plane. This vertical line is the lever arm for the off-center attached tension chords. During the simulation, it is equal to $l_{\mathrm{z}}=0.1 \mathrm{~m}$ if applicable. It can be imagined as a massless plate of zero thickness and infinite stiffness transferring the forces of the tension chords to the beam. 


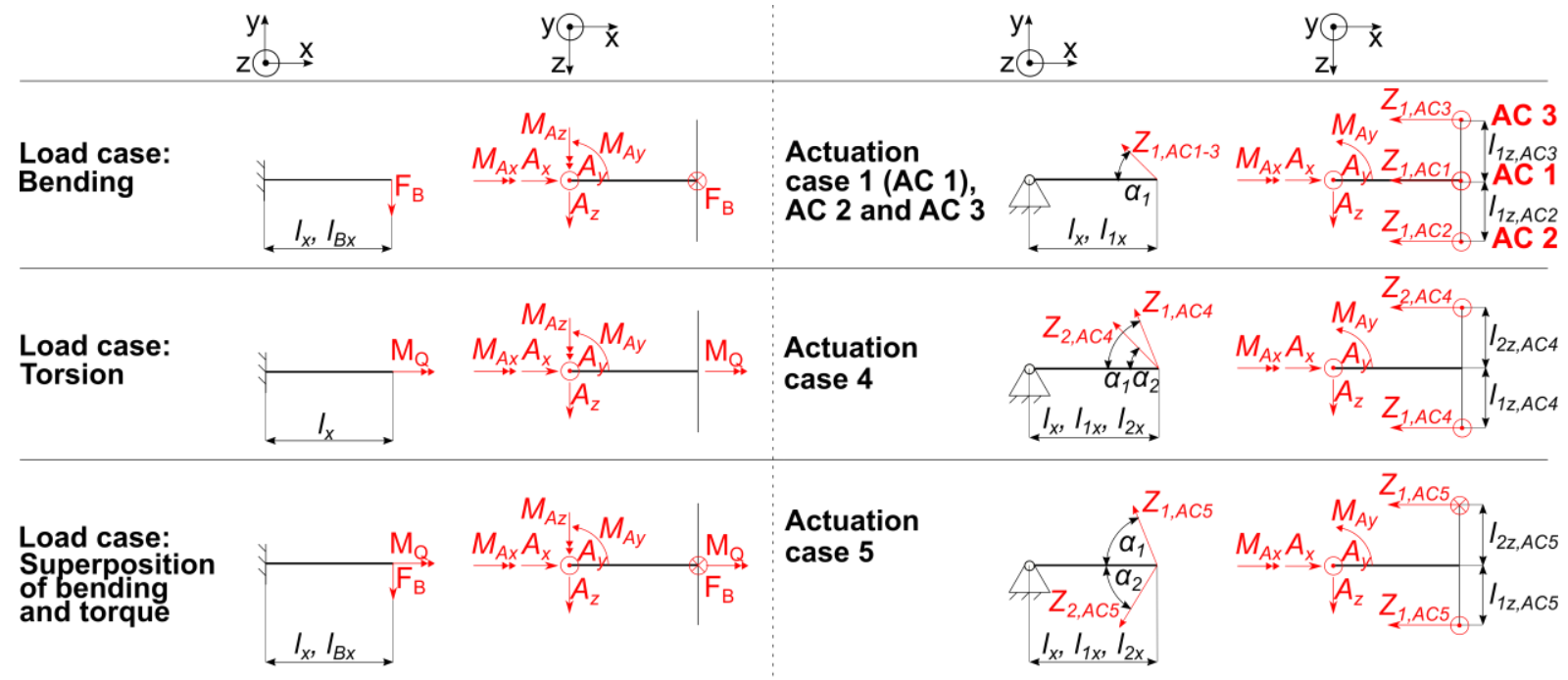

Figure 4. Load cases (left), actuation cases (AC, right) and support case fixed (left) and hinge (right) that are included in the investigation; The angles are given relative to the beam

The load cases shown for fixed support are also used for Actuation cases (AC) 1-5. Only one actuator is available for AC 1, 2 and 3. Depending on the case, the actuator is positioned in the middle or offcenter. With AC 2 and AC 3, torsion compensation is possible, but not with AC 1. In the superposition load case, bending must be compensated with AC 2 and AC 3 in order to ensure the target movement. Therefore, an additional tension chord is added in AC 4 and AC 5. These AC are also displayed in Figure 3B. The redundancy ensures the target movement and compensates torsion. During the simulation the values $F_{\mathrm{B}}=1000 \mathrm{~N}$ and $M_{\mathrm{Q}}=1000 \mathrm{~N} \mathrm{~m}$ are used. The absolute values of the angles are equal for the simulation to reduce the variation problem.

\subsection{Optimization}

The actuator forces are determined by optimization. The total volume of the beam to be minimized is defined as the objective function of the optimization. The objective functions are given in Equation (3) and Equation (4).

$$
\begin{aligned}
& f\left(Z_{1}\right)=V\left(Z_{1}\right), \min \left(\mathrm{f}\left(\mathrm{Z}_{1}\right)\right), \quad \mathrm{AC} 1, \mathrm{AC} 2 \text { and } \mathrm{AC} 3 \\
& f\left(Z_{1}, Z_{2}\right)=V\left(Z_{1}, Z_{2}\right), \min \left(\mathrm{f}\left(\mathrm{Z}_{1}, Z_{2}\right)\right), \text { AC } 4 \text { and } \mathrm{AC} 5
\end{aligned}
$$

When solving the function, the inserted force values have to fulfil an equality constraint given in Equation (5). The constraint results from the hinge support, i.e. $M_{\mathrm{Az}}$ equals zero.

$$
F_{B} \cdot l_{B x}=\left\{\begin{aligned}
Z_{1} \cdot \sin \left(\alpha_{1}\right) \cdot l_{1 x}, & \text { AC } 1, \text { AC } 2 \text { and AC } 3 \\
Z_{1} \cdot \sin \left(\alpha_{1}\right) \cdot l_{1 x}+Z_{2} \cdot \sin \left(\alpha_{2}\right) \cdot l_{2 x}, & \text { AC } 4 \text { and AC } 5
\end{aligned}\right.
$$

To find the global minimum of the function, GlobalSearch of MatLab's Optimization Toolbox is used. The solver GlobalSearch uses the nonlinear programming solver fmincon to find the global minimum. To put it in a simplified way, GlobalSearch uses the solver fmincon to find local minima for optimization problems. The local minimum found depends on the initial values of the optimization, i.e. the first values for the variable when the optimization is started. GlobalSearch uses several initial values to find various local minima. The function then finds smallest of those minima and returns the function input values for the global minimum.

\subsection{Discussion of assumptions and boundary conditions}

For the pilot study in this paper, we synthesized a system that is simple in comparison to the systems presented in Figure 2. We aim to evaluate the feasibility in terms of achievable weight savings for the loaded structure of the synthesized system. If weight cannot be saved for the synthesized system, it is unlikely that weight savings are possible for systems that are more complex. In real-life applications, 
the costs associated with the application of the principle also play an important role in determining the benefit of the principle besides the achievable weight savings. Additionally, we must consider the technical implementation of tension chording principle-based solutions. Notably, the weight reduction potential also depends on the technical realization of the system at hand.

The total weight of a system consists of the weight of the individual components. A weight reduction of one component can lead to an increase or decrease of weight for another component. This depends on how the elements are connected in the system. For example, reducing the weight of an excavator arm can also reduce the counterweight in the body. However, if the principle requires additional actuators in the system, the total weight increases again. In order to be able to make a statement on the total weight saving, we must know the technical specifications of all components. The investigations in this paper are therefore limited to the achievable weight saving of the structure as shown in Figure 3A.

The achievable weight saving of a structure is influenced by various factors. In addition to the material properties, the external loads and the loads introduced by the actuators are also important. The material properties mainly determine the mass of the system. Since Bartz et al. (2018a) already investigated how tension chords can compensate the bending caused by the weight of the system, it is not part of this investigation. The weight force caused by the mass is therefore not taken into account any further. Instead, two load cases and their superposition are investigated with straight bending and torsion. Only one load per load case is taken into account, i.e. one load causing bending, one load causing torsion, and their superposition, in order to limit the scope of the investigation. The loads are modelled as point forces on the system, area loads are not considered.

Considering the force balance of the system, loads that can be introduced by the actuators depend on several factors. The number of actuators determines how many different loads can be compensated, i.e. become zero for the system. However, without further factors, no conclusions can be drawn as to which loads can be minimized by the tension chords. Instead, the position of the actuators and the load application angle between the tension chords and the structure need to be considered as well. The investigation is limited to two actuators, which is assumed to correspond to the minimum number of actuators required to compensate for two load cases. Pauwels (1965) has found that the area of the load introduction is also important. He shows for the human locomotive system that muscles connected to the bone over a certain area lead to more favorable structural loads than point forces. Yet for this pilot study, actuator point forces are sufficient to further reduce the complexity of the example system by omitting actuator surface forces. In a previous study on minimizing bending by tension chords (Bartz et al., 2018a), the tension chords could only apply forces in one direction along their axis. In this study, the actuators are able to apply forces in both directions along their axis in this investigation. Thus, tension-compression actuators are included. The results might show in which case pure tension or compression actuators provide a benefit. In order to investigate the influence of the load application angle on the structure weight, the angle is varied.

The technical feasibility of the determined results may not be given depending on the application. In this case, the actuators play a role in combination with the structure and the occurring load cases. For example, the reaction time of the actuators can be too slow to minimize the occurring loads. Another example could be that the required load application angles cannot be achieved for the intended motion. These and other cases depend on the boundary conditions and require further investigations which exceed the scope of this pilot study.

\section{Results and discussion}

Within the scope of the investigation, the weight savings that can be achieved compared to a reference system are examined for different load cases, actuator positions and angles. The results of the simulation are shown in Figure 5. The relative volume expressed in percentage is plotted on the ordinate. It is calculated from the result of the volume optimization of the corresponding actuation case (AC) and load case divided by the volume resulting for the fixed support and same load case. The closer a value is to $100 \%$, the lower the volume savings compared to fixed support. On the abscissa, the angle of the tension chords according to Figure 4 is plotted. The angles were varied between $-90^{\circ}$ and $90^{\circ}$ in $1^{\circ}$ increments. For AC 4 and 5, the absolute values of the angles of the two tension chords are set equal in order not to reduce the size of the variation problem. Outliers can be identified which can be eliminated by improved 
optimization settings for these cases. No value is available for an angle of $0^{\circ}$ in all load and actuation cases, as the tension chords cannot fulfil the equality constraint in this case.

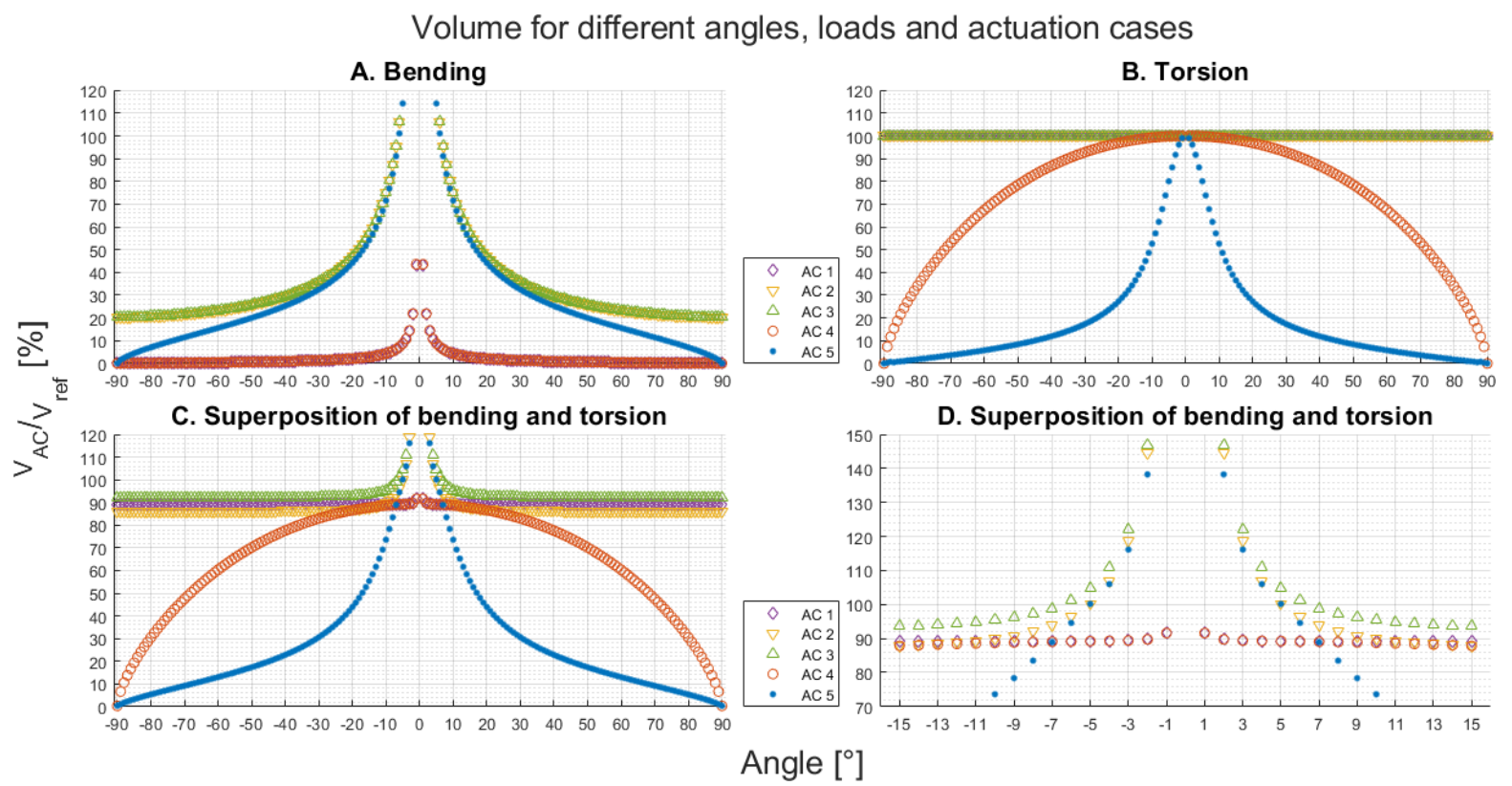

Figure 5. Relative volume resulting for actuation cases (AC) 1 to 5 , angles from $-90^{\circ}$ to $90^{\circ}$ and load cases bending (A), torsion (B), their superposition (C) and a detailed view on the superposition from $-15^{\circ}$ to $15^{\circ}$ (D)

A comparison of the various load cases and AC 1 to 3 shows the influence of the equality constraint (Equation 5) of the optimization and the angle between the tension chords and the beam on the results for the individual AC. In the torsion load case (Figure 5B), AC 1 is overlapped by AC 2 and 3 and they are all equal to $100 \%$. Theoretically, the off-centered positioning of the tension chord in AC 2 and 3 could reduce the torsion. If the torsion is compensated in one of these cases, the tension chord simultaneously causes a moment about the z-axis. However, the moment about the z-axis must be zero due to the equality constraint, so the constraint can only be met if the tension chord force is zero. A single tension chord used for torsion therefore cannot generate movement and compensate torsion at the same time when the bending is zero. On the other hand, as to be seen for the load case bending (Figure $5 \mathrm{~A}$ ), when the tension chords generate movement, they also introduce torsion and a bending torque into the system. Because of these extra moments, the volume of AC 2 and 3 is larger than the volume for AC 1. For the angles $-90^{\circ}$ and $+90^{\circ}$, only torsion is added to the total load when fulfilling the equality constraint by the tension chord of AC 2 and 3. This is possible because the tension chord force is pointing into the $y$-direction for these angles. If the absolute value of the angle is smaller than $90^{\circ}$, a part of the tension chord force points into the $\mathrm{x}$-direction which leads to a bending moment about the y-axis. The smaller the angle, the smaller the portion of the tension chord force acting in the y-direction which is needed to compensate the bending force. At the same time, the portion of the tension chord force acting in the $\mathrm{x}$-direction increases with a decrease of the absolute value of the angle. This increases the bending moment about the $y$-axis and leads to a curve which reminds of a $x^{-2}$-function. For small absolute values of the angle, the volume value calculated for the load case and fixed support is exceeded due to this bending moment. But the extra torsion moment introduced by the tension chord can also have a benefit, as to be seen for the superposition of the load cases (Figure 5C). AC 1 can only compensate bending as the tension chord force has no lever arm to the $\mathrm{x}$-axis. It serves as the reference value for the two other one-chord AC. As discussed, both AC 2 and 3 provide additional moments to the beam when fulfilling the equality constraint. Depending on the position of the tension chord, the torsion added by the tension chord either reduces (AC 2) or increases (AC 3) the torque the system is loaded with. As in the bending load case, the two AC are depending on the absolute value of the angle. Both curves have a small inclination towards the higher volume values between the absolute angle values $90^{\circ}$ and $45^{\circ}$. Starting from $45^{\circ}$ towards $0^{\circ}$ absolute value angle, the inclination increases. As in the bending case, volumes of 
AC 2 and 3 for angles close to zero exceed the volume calculated for fixed support and the same load cases. To conclude, using one tension chord only with the intention to compensate torsion is not promising. However, depending on its position it can be useful to reduce torque when mainly compensating bending. Especially for angles close to zero, an off-centered position of a single tension chord that is simultaneously used to generate a movement should be avoided. Instead, a centered position of the tension chord in which it does not influence on the torsion should be preferred. However, by adding a second tension chord some of the problems of using only one tension chord might compensate some of the additional moments one off-centered tension chord creates.

The influence of the angle and the equality constraint is also present for two tension chords as in AC 4 and 5. In AC 4, both tension chord forces point into the same direction (Figure 4). In the torsion load case, the sign of one tension chord force must be negative while the other must be positive to ensure the equality constraint and to compensate the torque. Yet, this leads to a bending moment about the y-axis. With decreasing absolute value of the angle, the bending moment becomes higher in relation to the torsion that can be reduced at the same time. Therefore, a decreasing amount of torsion is reduced for angles closer to zero. In contrast, AC 5 can reduce most of the torsion. In this case, the x-component of the chord forces points in the same direction, while the y-components point in the opposite direction. For torsion, this means that the bending about the y-direction introduced by one chord is compensated by the bending introduced by the other chord. Therefore, the volume only depends on normal forces caused by the tension chords. While the angles get closer to zero, it is beneficial to reduce a decreasing amount of torsion as the normal forces become larger. For the load case bending, the directions of the tension chord forces of AC 4 are more beneficial as the ones of AC 5. The volume values of AC 4 overlap the values of AC 1. For this load case, the bending about the y axis is compensated in actuation case 4, while it is added in AC 5. Yet, the bending about the y axis introduced by AC 5 is smaller for most angles as in AC 2 and 3. For angles with absolute values of less than $7^{\circ}$ results for AC 4 are better than for AC 5, as can be seen in Figure 5D. Another aspect that is visible in Figure 5D is the congruence of values for AC 4 and $\mathrm{AC} 1$ for angles of absolute value 10 or smaller. For these small angles, compensation of torsion is not beneficial in AC 4. To conclude, the results show that the arrangement of the tension chords plays a decisive role for the benefit of the principle. Tension chords can reinforce each other or, depending on the constraints, fail to achieve their purpose and, in the worst case, increase the total load. For all angles and $\mathrm{AC} 4$ and 5, tension-compression actuators are necessary to compensate torsion, thus tension actuators as in Bartz et al. (2018a) would not work for the given AC. Further research could investigate whether another tension chord can bring advantages.

With the investigations we have shown that volume of the structure can be saved under torsion load with the tension chording principle. For the evaluation of the lightweight design use, the boundary conditions must become more specific. We cannot evaluate it in general terms, which AC is particularly good regarding further influencing factors with a generic system as used in this paper. For this reason, we must apply the tension chording principle to a more specific use case and evaluate it based on the boundary conditions and requirements.

\section{Conclusion}

Previous work has shown the benefits of tension chording as a lightweight design principle for structures subject to bending loads. In this paper, we make a pilot study on using the principle for torsion loaded structures. We show that the tension chording principle can also lead to volume savings in this case. However, the savings for torsion reduction are not as high as for bending reduction and - as identified for bending before - depend on both the actuator position and the number of actuators. In addition, the results for torsion are more sensitive to the angle between the actuators and the beam compared to the results generated for bending. The system is a basic example with many limiting assumptions, which do not allow an absolute statement on the lightweight benefit yet. Statements that are more general would only be possible when varying a large number of influencing factors, which would become a complex and time-consuming task. This calls for a design of experiment approach, which needs to be triggered by more concrete assumptions and limitations related to a technical application. So as the next step, we are going to apply the principle to systems with concrete boundary conditions and specifications. With the specific benefit for these systems, we can make assumptions about the benefit for similar systems. These 
assumptions will then support the general statement on the potential of tension chording as a lightweight design principle. For a designer, this pilot study can serve as an impulse for new lightweight design concepts of kinematic chains. Future investigations and applications of the principle might fortify the benefits identified in this pilot study also for real world applications.

\section{References}

Ananthanarayanan, A., Azadi, M. and Kim, S. (2012), "Towards a bio-inspired leg design for high-speed running", Bioinspiration \& biomimetics, Vol. 7 No. 4, p. 46005. https://dx.doi.org/10.1088/1748-3182/ 7/4/046005

Bartz, M., Brand, H. and Bender, B. (2018a), "Examing lightweight design potential of the human musculoskeletal system by using the example of an articulated arm robot", Book of Abstracts: 1. Symposium for Lightweight Design in Product Development, Zürich, Switzerland, June 13-15, 2018.

Bartz, M. et al. (2018b), "Development of a bioinspired approach for the design of kinematic chains", Design 2018: proceedings of the 15th International Design Conference, Dubrovnik, Croatia, May 21-24, 2018, The Design Society, Glasgow, pp. 975-984. https://dx.doi.org/10.21278/idc.2018.0330

Bartz, M., Uttich, E. and Bender, B. (2019), "Transfer of lightweight design principles from the musculoskeletal system to an engineering context”, Design Science, Vol. 5, p. e19. https://dx.doi.org/10.1017/dsj.2019.17

Degischer, H.-P. and Lüftl, S. (2009), Leichtbau. Prinzipien, Werkstoffauswahl und Fertigungsvarianten, WileyVCH (Wiley Online Library), Weinheim. https://dx.doi.org/10.1002/9783527628247

Klein, B. (2013), Leichtbau-Konstruktion. Berechnungsgrundlagen und Gestaltung, Springer, Wiesbaden. https://dx.doi.org/10.1007/978-3-658-02272-3

Klug, S. et al. (2005), "Design and Application of a 3 DOF Bionic Robot Arm", AMAM 2005, Illmenau, Germany, September 25-30, 2005.

Mattheck, C. (1997), Design in der Natur, Rombach GmbH \& Co Verlagshaus KG, Freiburg.

Möhl, B. (2003), “A Composite Drive with Separate Control of Force and Position”, Proc. of the 11th International Conference on Advanced Robotics 2003 in Coimbra (ICAR 2003), IEEE Robotics and Automation Society, pp. 1606-1610.

Neilson, P.D. (1993), "The problem of redundancy in movement control. The adaptive model theory approach." Psychological Research, Vol. 55 No. 2, pp. 99-106. https://dx.doi.org/10.1007/BF00419640

Pauwels, F. (1965), Gesammelte Abhandlungen zur funktionellen Anatomie des Bewegungsapparates, SpringerVerlag, Berlin, Heidelberg, New York.

the Press and Information Office of the Federal Government (2018), German Sustainable Development Strategy, Druck- und Verlagshaus Zarbock GmbH \& Co. KG, Berlin.

Reuschel, D. (1999), Untersuchung der Faseranordnung natürlicher Faserverbunde und Übertragung der Ergebnisse auf technische Bauteile mit Hilfe der Finite-Elemente-Methode, [PhD Thesis], Universität Karlsruhe.

Richard, H.A. and Kullmer, G.K. (2014), Biomechanik: Grundlagen und Anwendungen auf den menschlichen Bewegungsapparat, Springer-Verlag, Wiesbaden. https://dx.doi.org/10.1007/978-3-8348-8611-8

Scott, S.H. (2004), "Optimal feedback control and the neural basis of volitional motor control", Nature Reviews Neuroscience, Vol. 5 No. 7, pp. 532-546. https://dx.doi.org/10.1038/nrn1427

Uttich, E., Bartz, M. and Bender, B. (2019), "Factors preventing the use of a lightweight design workflow that is inspired by the human locomotive system", Proceedings of the Design Society: International Conference on Engineering Design, Cambridge University Press, Vol. 1 No. 1, pp. 2705-2714. https://dx.doi.org/10.1017/ dsi.2019.277

VDI (2019), VDI 6220-1: Biomimetics - Fundamentals, conception and strategy, Berlin.

VDI (2017), VDI 6224-3: Biomimetics - Integrated product development process for biomimetic optimisation, Berlin.

Völkl, H., Franz, M. and Wartzack, S. (2017), "Topologieoptimierung mit transversal isotropem Materialmodell - Produktentwickler auf der Suche nach optimaler Geometrie für Faser-Kunststoff-Verbunde", DFX 2017: Proceedings of the 28th Symposium Design for X, 4-5 October 2017, Bamburg, Germany, The Design Society, pp. 203-214.

Weiner, S. and Wagner, H.D. (1998), "The Material Bone: Structure-Mechanical Function Relations", Annual Reviews Material Science 1998, Vol. 28, No. 1, pp. 271-298. https://dx.doi.org/10.1146/annurev.matsci.28.1.271

Wiedemann, J. (2007), Leichtbau. Elemente und Konstruktion, Springer (Klassiker der Technik), Berlin, Heidelberg, New York. https://dx.doi.org/10.1007/978-3-540-33657-0

Witte, H. et al. (2000), "Konstruktion vierbeiniger Laufmaschinen anhand biologischer Vorbilder". Konstruktion, Vol. 9, pp. 46-50.

Witzel, U. and Preuschoft, H. (2005), "Finite-element model construction for the virtual synthesis of the skulls in vertebrates: case study of Diplodocus". The anatomical record. Part A, Discoveries in molecular, cellular, and evolutionary biology, Vol. 283, No. 2, pp. 391-401. https://dx.doi.org/10.1002/ar.a.20174 